\title{
A HYDROGEN PURIFICATION AND STORAGE SYSTEM USING METAL HYDRIDE
}

\author{
Shinichi Miura and Akitoshi Fujisawa, Kobe Steel, Ltd., Kobe, Japan \\ Masayoshi Ishida, University of Tsukuba, Tsukuba, Japan
}

a Kobe Steel, Ltd., 1-5-5, Takatsukadai, Nishi-ku Kobe, Hyogo 651-2271, Japan

b University of Tsukuba, Tsukuba, Japan

\begin{abstract}
This work is to develop a new hydrogen purification and storage system for daily start and stop (DSS) operations. The new system enables us to minimize emissions of carbon dioxide by using compact and highly efficient fuel cells. The new system first removes carbon monoxide, which is poisonous to metal hydride, from reformed gas by using a special carbon monoxide adsorbent. After removing carbon monoxide, the reformed gas is introduced to a metal hydride bed to purify and store hydrogen. Some $100 \mathrm{NL} / \mathrm{h}$ Laboratory scale apparatus was operated in daily start and stop operations for 100 cycles for a total of 150 hours with quite good efficiency. The new process has achieved an $83 \%$ hydrogen recovery ratio in one-month DSS operations.
\end{abstract}

Keywords;

Hydrogen, purification, storage, Metal hydride, DSS

\section{Introduction}

The coexistence of energy supply and environmental protection is said to be a major goal for the industrial society of the 21st century. In the 20th century, a social system was established that placed priority was on the efficiency and the cost, by mainly consuming oil as an energy source. The existing social system is built by assuming both large production and large consumption, and also setting a goal to minimize investment in infrastructure and energy costs. It is important to develop fundamental and cross functional technology as a countermeasure against global warming. We must aim to not only save energy in our existing society, but also at reform our social and economic systems in order to decrease carbon dioxide emissions drastically. Utilization of hydrogen energy is promising in view of energy system reformation. In this situation above, hydrogen production and supply technology development will become important. We want to discuss especially hydrogen purification and supply process.

The only available hydrogen purification technology for use on a small scale as of 2010 is hydrogen Pressure Swing Adsorption (PSA) technology. This has long been used in the oil refining process on an industrial scale. This technology eliminates impurity gases from a mixed gas of hydrogen, water, carbon dioxide, carbon monoxide, and methane using adsorbent. Continuous operation is the basic operational mode since it is converted from the industrial oil refining process. Internal layer diffusion of impurities, which are desirable to be adsorbed and eliminated from gas flow, occurs within the adsorption tower when the process is stopped, causing lower purity of the product at next start of the process. And also, a rapid start disturbs the impurity gas within the adsorption tower, also lowering the purity of the hydrogen gas. As is discussed above, it is difficult to use the hydrogen purification process to supply pure hydrogen to the energy industry, in which electricity demand is low at night. Further, it is difficult to 
establish an infrastructure since it will not be easy to operate the system at the early stage of its application. There are needs for a compact and high-performance hydrogen purification process.

This study describes a new hydrogen purification and storage system that can be applied to a compact facility for making and purifying hydrogen, and that can be applied to daily start and stop operations. Thus, the possibility of having a power generation system that minimizes carbon dioxide emissions per unit energy by virtue of the high efficiency of the fuel cell even for small size and low load operations will be verified. Its ability to handle changes in demand and supply will also be verified.

\section{Experimental setup}

\subsection{Process background}

Metal hydride has a high storage volume efficiency. Therefore, research and development of a new metal hydride for use in vehicles is proceeding in order to improve their storage-weight efficiency. On the other hand, if no poisonous substances are included, metal hydride can be used as a gas purification and storage material that selectively stores hydrogen when a mixed gas is supplied to it. As far as a fixed-type of apparatus is concerned, the existing AB5 type of metal hydride can be used. Metal hydride absorbs and releases hydrogen in an equilibrium process according to the designed alloy temperature and pressure. When it is maintained at an equilibrium temperature it will not affect the hydrogen maintaining pressure, and it keeps the absorbed amount of hydrogen. On the other hand, when the pressure in a system falls because hydrogen is extracted, the metal hydride releases hydrogen in order to keep the equilibrium pressure. By making the best of this characteristic, a power generation system with a passive adjustment of supply and demand can be realized if a fuel cell is connected to the metal hydride that stores pure and pressurized hydrogen. In other words, a system can be made where the consumed hydrogen is compensated for by the metal hydride.

The University of Tsukuba has reported that carbon monoxide in reformed gas is absorbed on the surface of metal hydride and it prevents hydrogen from being absorbed. It is necessary to eliminate the carbon monoxide to achieve a hydrogen purification process using a metal hydride. [1] [2] [3] As a result of a joint investigation conducted by Kobe Steel and the University of Tsukuba, a new process is proposed. First, this method thoroughly eliminates carbon monoxide using an adsorption material that strongly selects carbon monoxide, and second it introduces a mixed gas to a metal hydride that purifies

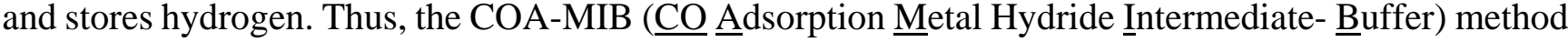
was established.

Figure 1 shows a schematic diagram of this system.

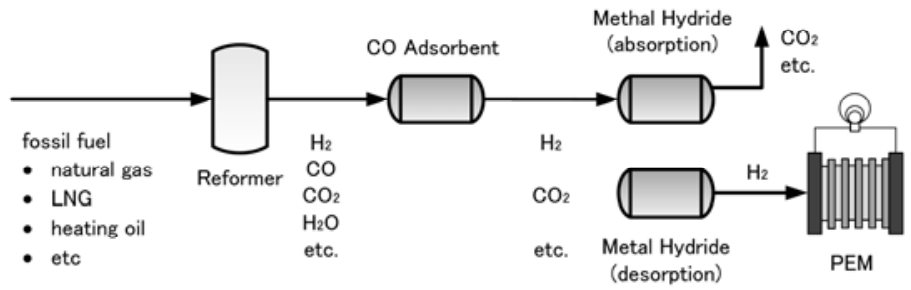

Fig. 1 Schematic diagram of the COA-MIB system

The COA-MIB system, which utilizes a metal hydride as a selective purification material of hydrogen, has the following merits as a hydrogen purification system.

1) Carbon monoxide will not be mixed into the pure hydrogen that is supplied to the fuel cell since it is 
completely eliminated by the carbon monoxide adsorption tower prior to supply.

2) Hydrogen is selectively separated from other impurities and purified as an adsorption material. Therefore, selectivity can be kept high, loss is low, and a high recovery rate of hydrogen can be achieved.

3) In the existing Pressure Swing Adsorption (PSA) system, reverse mixing occurs within the adsorption layer when the process is stopped since it eliminates impurities with a weak adsorption force. However, the proposed system has a higher start and stop performance and a lower impurity loss, resulting in higher operational capability since it separates hydrogen selectively.

4) Hydrogen is once stored in a metal hydride, and then released depending on demand. This makes flexible operations possible corresponding to starts and stops, load changes, and operation stoppage at night, and allows hydrogen to be immediately supplied after start-up. Operating reformer equipment and supplying pure hydrogen are independent processes, and therefore it can work independently of the reformer equipment.

5) The following two types of operation are available. One is to operate a small reformer steadily both in the daytime and at night, accumulating hydrogen in a metal hydride, and using hydrogen in a concentrated manner in the daytime. The other is to operate a large reformer only in the daytime to cope with demand, while stopping operations at night.

6) The purification container can also be used as a storage container. Therefore, there is no need for a large buffer for compressed hydrogen.

\subsection{Test apparatus}

The COA-MIB process was validated in order to confirm its ability to produce pure hydrogen. Methanol was selected as the raw material, steam was reformed, and we used $\underline{\text { CO }}$-Pressure $\underline{\text { Vacuum }}$ Swing Adsorption (CO-PVSA), which eliminates carbon monoxide with its selective adsorbent. It is known that $\mathrm{Cu}(\mathrm{I})$ binds to $\mathrm{CO}$ selectively. The adsorbent used in the process consists of an activated alumina carrier, which has an appropriate pore size distribution and uses an impregnated carbon and copper compound $\left(\mathrm{CuCl}_{2}, \mathrm{CuCl}\right)$ as the active chemical adsorption species.[4] We also used the COA-MIB laboratory test apparatus consisting of MIB that continuously produces high-purity hydrogen by MH. A daily start and stop operation (DSS) test was carried out in the experiment to produce pure hydrogen.

The test apparatus was able to produce 100 NL of hydrogen per hour.

Figure 2 shows the process flow diagram of the experimental apparatus. Figure 3 shows photographs of the experimental apparatus.

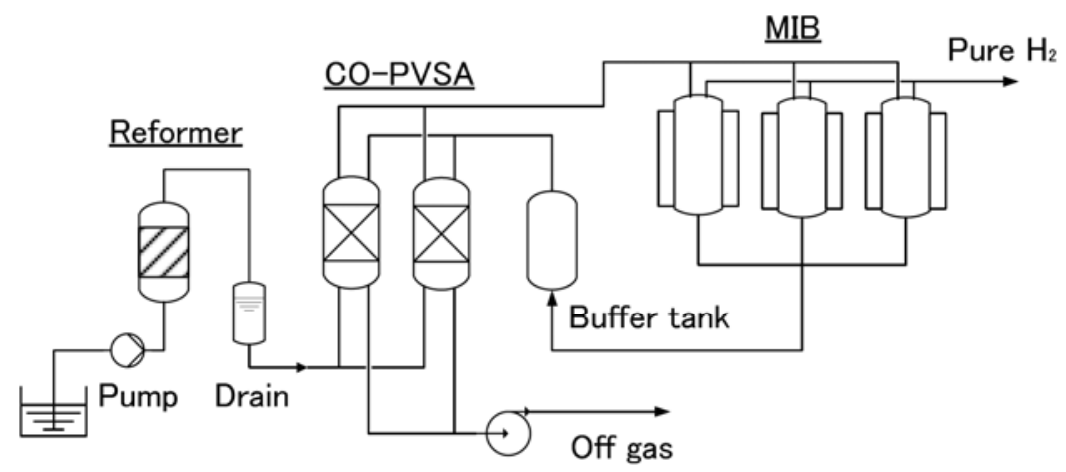

Fig. 2 Process flow diagram of COA-MIB Laboratory’s continuous operation system 


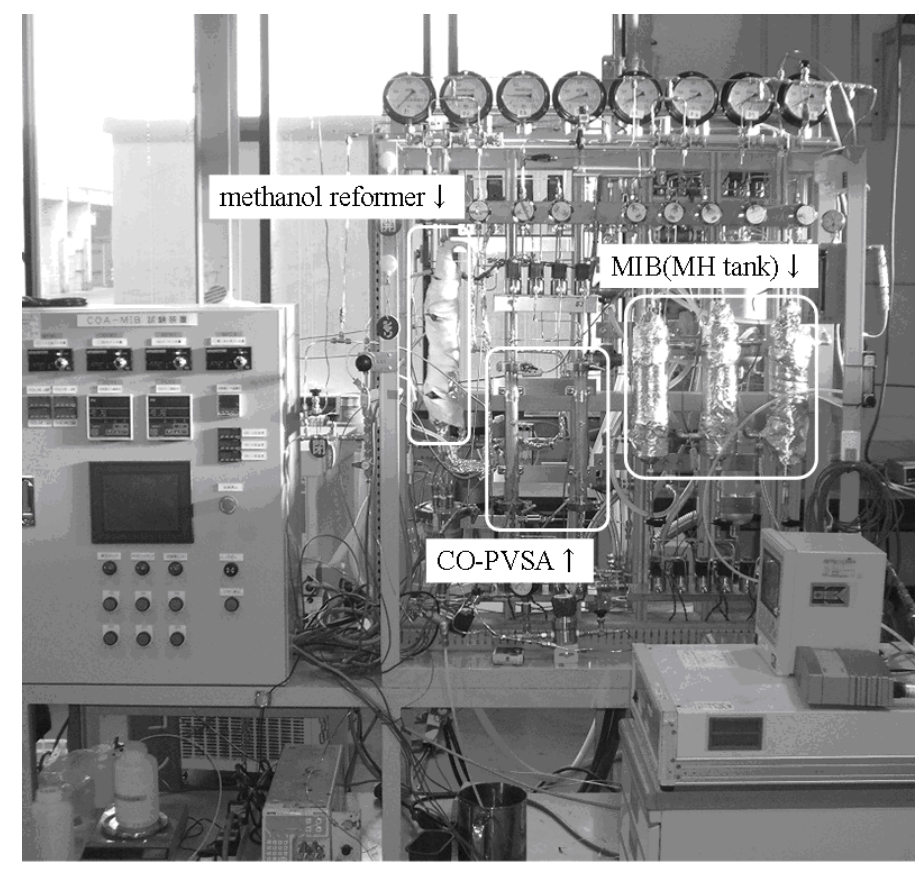

Fig. 3 Photograph showing COA-MIB Laboratory’s continuous operation system

\subsection{Methanol Reformer}

Methanol and water were sent to the steam reformer at a flow rate of $1.533 \mathrm{~g} / \mathrm{min}$ by a transfer pump. The reformation reaction was conducted at $235^{\circ} \mathrm{C}$ and $0.9 \mathrm{MPag}$. The $\mathrm{S} / \mathrm{C}$ ratio of raw material, methanol and water was 2.0. The reformer tube had a diameter of $46 \mathrm{~mm}$, and the filling volume of the catalyst was 450 cc. The catalyst for methanol steam reformation was MDC-3 (Süd-Chemie Catalyst Japan), and $540 \mathrm{~g}$ of MDC-3 was used. MDC-3 consists of $\mathrm{CuO}, \mathrm{ZnO}$, and $\mathrm{Al}_{2} \mathrm{O}_{3}$. Humidity was eliminated from the obtained reformed gas and sent to CO-PVSA.

\subsection{Water and CO elimination}

The CO-PVSA was of a two-tower type. The adsorption tower was filled with activated alumina adsorbent to completely eliminate the humidity, and the adsorption tower was filled with selective adsorbent of carbon monoxide to eliminate carbon monoxide. The tower was $19.4 \mathrm{~mm}$ in diameter. The filling volume of activated alumina was 24.2 cc, and 70 cc of selective adsorbent of carbon monoxide was used. The selective adsorbent of carbon monoxide used in this process was co-developed by Kobe Steel and Kansai Coke and Chemicals Co., Ltd. and it is an adsorbent based on porous alumina supporting $\mathrm{Cu}(\mathrm{I})$. Chemical adsorption of carbon monoxide occurs on the supported $\mathrm{Cu}$ (I). [4] The actual operation adsorption capacity of carbon monoxide is around 7 times in compare with that of zeolite, and the selective adsorption of carbon monoxide occurs, which is a characteristic of this process. The designed capacity was 7 times This adsorbent was confirmed to be capable of completely eliminating a small amount of carbon monoxide until a breakthrough occurs. It was clarified that there is very little leakage in the carbon monoxide elimination process by adsorption, until the lower limit of detection occurs, if proper regeneration is conducted. [5][6]

We switched between pressurized absorption and de-pressurized regeneration every 5 minutes in the adsorption tower. The pressure was set to $0.85 \mathrm{MPag}$ for adsorption. We supplied off-gas (mainly carbon 
dioxide) as the sweep gas from $\mathrm{MH}$ stored in the buffer tank during de-pressurized regeneration. De-pressurization was conducted with a vacuum pump. Gas whose humidity and carbon monoxide were to be eliminated by CO-PVSA was supplied to MIB in the down-flow direction.

\subsection{Metal hydride intermediate buffer}

The MIB, metal hydride intermediate buffer, was of a three-tower type. The pressure of hydrogen absorption in MIB was 0.8 MPag. The hydrogen concentration in feed gas was around 70\%.

The process of hydrogen absorption and purification, de-pressurized purge, and hydrogen releasing was switched among the three towers enabling continuous purification and release. $\mathrm{MH}$, whose alloy composition and mixing ratio was adjusted to have an equilibrium pressure of $0.2 \mathrm{MPa}$ at $20^{\circ} \mathrm{C}$, was used as the metal hydride.

The following metal hydride that is capable of hydrogen purification was manufactured:

AB5-type metal hydride whose hydrogen equilibrium pressure is adjusted to be $0.2 \mathrm{MPag}$ at $20^{\circ} \mathrm{C}$. (MmNi3.99Co0.6Mno.36Alo.05)

The metal hydride was supplied by Japan Metals \& Chemicals Co,Ltd. The hydrogen absorption performance of this metal hydride is shown as a PCT curve in Fig.4.

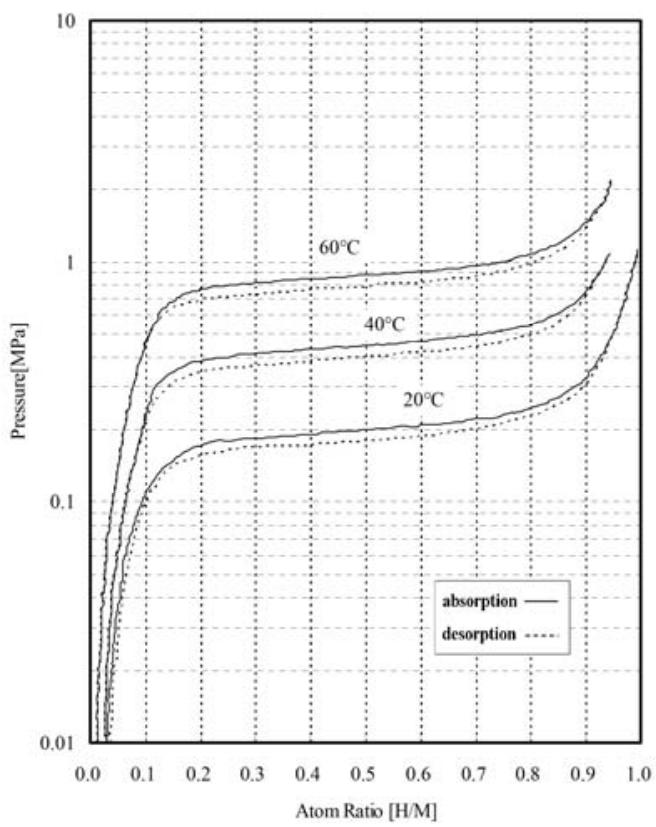

Fig. 4 PCT curve of $\mathrm{MH}$

The $\mathrm{MH}$ container was $46 \mathrm{~mm}$ in diameter and it had a water jacket to control the process temperature. We circulated cold water at $20^{\circ} \mathrm{C}$ in it during hydrogen absorption to control heat generation due to hydrogen absorption, and also circulated hot water at $80^{\circ} \mathrm{C}$ to compensate for the heat lost in the heat absorption reaction during hydrogen release. The cold water $20^{\circ} \mathrm{C}$ and hot water $80^{\circ} \mathrm{C}$, used for $\mathrm{MH}$ container, were designed as a low quality waste heat which will be available in many situations.

The period of hydrogen absorption with cooling was $30 \mathrm{~min}$, the period of hydrogen release with heating was $30 \mathrm{~min}$, and the third tower was waiting for absorption with cooling. The MIB operation cycle was 1.5 hours. 
The equilibrium hydrogen pressure of metal hydrides varies depending on their alloy composition. [7][8] This means that a metal hydride suitable for the actual process can be designed by changing its alloy composition. Absorption and release of hydrogen, that is reactions of a hydrogen compound generation and dehydrogenation reaction, are a heat generation reaction and a heat absorption reaction respectively. Therefore, it is essential to control the temperature of the metal hydride filling layer.[9][10][11][12]

\subsection{Analyzers and Materials}

The COA-MIB system purifies hydrogen from the pressurized reform gas and releases pure hydrogen in a heating and depressurizing process.

The amounts of reforming gas, outlet gas of CO-PVSA, and off gas of the MIB system formed were measured as follows:

Hydrogen concentration: Thermal conductivity gas analyzer ZAF-3 (0-100\%) Fuji electric systems Carbon monoxide concentration: NDIR VIA510-CO (0-1/5/10/15\%) Horiba Carbon monoxide concentration: NDIR VIA510-CO (0-100/200/500/1000 ppm) Horiba Carbon dioxide concentration: NDIR VIA510-CO $2(0-5 / 10 / 25 / 50 \%)$ Horiba The purity of hydrogen in the release process was measured with GC-8-APF (a product of Shimadzu Corporation) every 10 minutes.

\section{Experimental results and discussion}

\subsection{Overall results}

The operation was conducted for 7 hours every day in DSS mode. Overall, the experiment was continued for 100 cycles over 28 days and the operational time was 150 hours. The accumulated data obtained from the pure hydrogen production test for 28 days is shown in Table 1 . The recovery rate of hydrogen, which is the ratio of obtained pure hydrogen to total hydrogen included in the reformed gas, reached 83\%.The hydrogen recovery ratio during the 100 cycles is shown in Fig. 5.

The hydrogen purification rate is defined by the following equation:

Amount of hydrogen absorption

$$
\text { Hydrogen recovery ratio = ---------------------------------------------- } \times 100
$$

Amount of hydrogen put into the system

It was confirmed with this result that there is no crucial deterioration in the hydrogen absorption rate during the 100 cycles, showing the feasibility of this process in the laboratory.

Table 1. Accumulated data for 100 cycles of operation of the COA-MIB Laboratory system

\begin{tabular}{|c|c|c|}
\hline $\begin{array}{c}\text { Hydrogen in reforming } \\
\text { gas }\end{array}$ & $\begin{array}{c}\text { Hydrogen in CO-PVSA } \\
\text { outlet gas }\end{array}$ & $\begin{array}{c}\text { Hydrogen in MIB } \\
\text { outlet gas }\end{array}$ \\
\hline $14.8 \mathrm{Nm}^{3}$ & $13.9 \mathrm{Nm}^{3}$ & $12.3 \mathrm{Nm}^{3}$ \\
\hline $\begin{array}{c}\text { Recovery } @ \\
\text { CO-PVSA }\end{array}$ & Recovery @ MIB & $\begin{array}{c}\text { Total hydrogen } \\
\text { recovery }\end{array}$ \\
\hline $94 \%$ & $88 \%$ & $83 \%$ \\
\hline
\end{tabular}




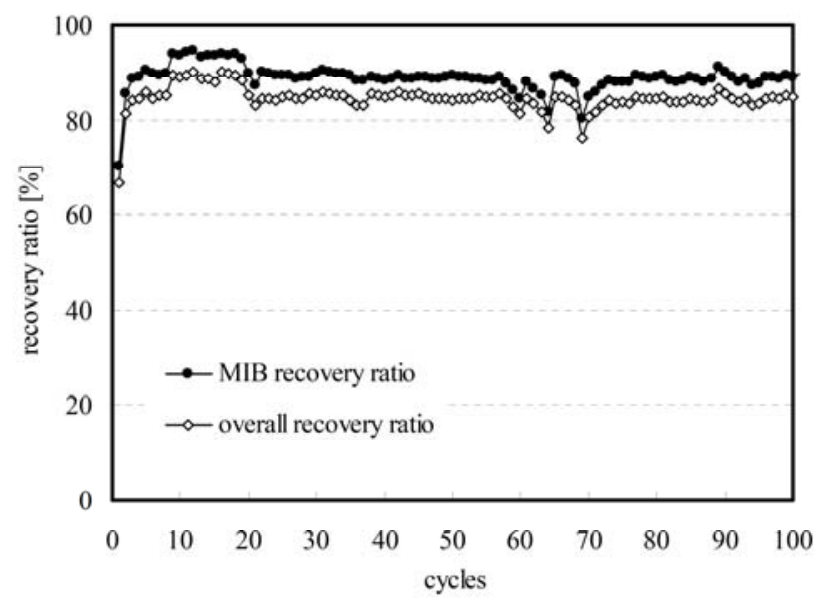

Fig. 5 Hydrogen recovery ratio during 100 cycles

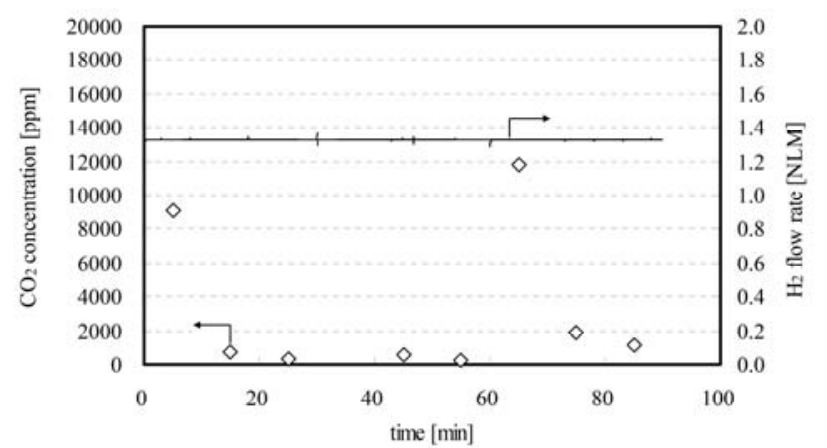

Fig. 6 Purity of product gas

\subsection{Product gas purity}

Figure.6 shows the relationship between the flow rate of the obtained pure hydrogen and the measured concentration of carbon dioxide. Impurities other than carbon dioxide that may intrude, such as methane or carbon monoxide, were not detected, indicating that the only impurity is carbon dioxide.

The switching was done every 30 minutes, and it was confirmed that carbon dioxide intrudes by about $1 \%$ as an impurity immediately after the switching. This is considered to be because the residual carbon dioxide in the container leaks since it is not expelled completely in the purging process. On the other hand, the concentration becomes lower than 2000 ppm 15 minutes after release initiation.

It is considered that carbon dioxide as an impurity will not cause any serious problems with fuel cell power generation conducted by a dead-end type of pure hydrogen fuel cell since purging is properly performed in the stack. On the other hand, the purity may not satisfy the ISO standards if the equipment is used as a pure hydrogen production facility for a hydrogen station. However, the present experimental results were obtained from a three-tower type MIB, and further improvement can be expected. For instance, adopting a four-tower type MIB will make it possible to introduce a purge process in the container by product gas, and this will be a very promising way to increase purity. Nonetheless, it was confirmed that carbon monoxide that may cause crucial damage to the fuel cell had been completely eliminated, and further investigation on the actual application of this system should be considered.

\section{Conclusions}


We have developed and demonstrated a new hydrogen purification and storage system for daily start and stop operations. The hydrogen produced by this system is free from carbon monoxide because it is eliminated at the first unit of this system.

The 100 NL/h Laboratory scale apparatus was operated in daily start and stop operations for 100 cycles for a total of 150 hours with adequate efficiency. Hydrogen absorption control for metal hydride were operated with $20^{\circ} \mathrm{C}$ and $80^{\circ} \mathrm{C}$ water circulation which is designed as a low quality heat. The new process achieved a hydrogen recovery ratio of $83 \%$ in one-month DSS operations. The results showed that there is no crucial deterioration in the hydrogen absorption rate during 100 cycles, showing the feasibility of this process in the laboratory.

The new system enables us to minimize emissions of carbon dioxide by using compact and highly efficient fuel cells as a part of smart community technologies. We would like to investigate the system further and help to build a new system. Active promotion of this development will help to enhance the technology and thus lead to a reformation of energy economics.

\section{Acknowledgement}

This work was supported by the Greenhouse Gas Mitigation Technology Development Program of MOE Japan. [13]

\section{References}

[1] M. Ishida, Passive Load Following Method by Purifying Hydrogen from Reformed Gas with Metal Hydride for Fuel Cell, IEEJ 2001; vol. 121-B, No. 8, 1036-1043

[2] R. Sasaki, M. Ishida, Effect of CO in Reformed Gas on Fluorinated Metal Hydride, IEEJ-B 2004 vol. 124, No. 7,965-970

[3] Y. Taniguchi, M. Ishida, Hydrogen Purification Method from Reformed Gas Containing High Concentration of CO by using Metal Hydride, IEEJ-B 2006,vol. 126-B, No. 12, 1267-1274

[4] F. Kasuya, T. Tsuji, High purity Co gas separation by pressure swing adsorption, Gas Separation \& Purification 1991,Vol.5,242-246

[5] S. Miura, et al., CO removal process from PEFC application, Conference of HESS Japan, 2004, 267-270

[6] S. Miura, 'CO Removal Process Development from Hydrogen Gas' The Journal of fuel cell technology, 2008, vol. 8, No. 1, 135-138

[7] T.Gamo, Y.Morisaki, et al. Life properties of Ti---Mn alloy hydrides and their hydrogen purification effect, J.less-common Met., 1983, vol.89, Issue 2, 495-504

[8] M.T.Hagström, et al., Metal hydride hydrogen storage for near-ambient temperature and atmospheric pressure applications, a PDSC study, IJHE, 1995, vol.20, No.11, 897-909

[9] A. Fujisawa, S. Miura, Hydrogen production method using CO adsorbent and Metal Hydride (Japanese) , 2008, Conference of SCEJ, M315

[10] A. Fujisawa, S. Miura, et al. 'Development of new hydrogen purification and storage system for PEFC using a special carbon monoxide adsorbent and metal hydride' Conference of the JIE, 2009,252-253

[11] A. Fujisawa, S. Miura, et al. 'Development of new hydrogen purification and storage system using a special carbon monoxide adsorbent and metal hydride.' Conference of HESS Japan, 2009, 79-81

[12] S. Miura, 'New hydrogen gas purification and storage system using metal hydride.' The Journal of 
fuel cell technology, 2009,vol. 9, No. 2,108-112

[13] S. Miura, A. Fujisawa, et al. Official report in trust MOE, Japan. Greenhouse Gas Mitigation Technology Development Program 2009.' 'Hydrogen purification \& storage system for DSS operation technology development.' 